\section{Prevalência da síndrome de burnout em trabalhadores de enfermagem de um hospital de grande porte da Região Sul do Brasil}

\author{
Prevalence of burnout syndrome in nursing staff \\ in a large hospital in south of Brazil
}

\author{
${ }^{1}$ Faculdade de Medicina, \\ Universidade do Sul de Santa \\ Catarina, Tubarão, Brasil. \\ Correspondência \\ F. R. L. Magajewski \\ Faculdade de Medicina, \\ Universidade do Sul de Santa \\ Catarina \\ Av. José Acácio Moreira 787, \\ C. P. 370, Tubarão, SC \\ 88704-900, Brasil. \\ flavio.magajewski@unisul.br
}

\section{Abstract}

Burnout syndrome is a chronic adaptive disorder that affects workers and is characterized by three forms of psychological distress: emotional exhaustion, depersonalization, and a sense of low personal fulfillment. A cross-sectional quantitative design was used to apply questionnaires including socio-cultural data and the Maslach Burnout Inventory in 151 nurses and nurse technicians, representing $54.1 \%$ of this professional category in a large general hospital in Tubarão, Santa Catarina State, Brazil. Mean scores were: emotional exhaustion (17), depersonalization (7.79), and low personal fulfillment (36.6). According to the criterion proposed by Ramirez et al., no cases of burnout were diagnosed. According to the criteria by Grunfeld et al., 35.7\% of the interviewees displayed burnout. The standard profile of workers with burnout identified in the study was: nurse technicians, female gender, age 26-35 years, married, with no children, and with five or more years in the profession. Hospital wards or areas with the highest proportion of nursing staff with burnout were the grouped hospital sectors (42.6\%), ICU (25.9\%), and neonatal ICU (18.5\%). There was no statistically significant association between ward or hospital area and burnout.

Professional Burnout; Nursing Services; Occupational Health
Davi de Souza Moreira 1

Renata Faverzani Magnago ${ }^{1}$

Thiago Mamôru Sakae 1

Flávio Ricardo Liberali Magajewski 1

\section{Introdução}

O termo burnout foi utilizado pela primeira vez em 1974, mencionado pelo psicólogo Herbert J. Freudenberger 1, que descreveu um quadro observado em jovens trabalhadores de uma clínica de dependentes de substâncias químicas na cidade de Nova York, Estados Unidos. Esses funcionários reclamavam que já não conseguiam ver os pacientes como pessoas que necessitavam de ajuda, uma vez que estes não se esforçavam em seguir o tratamento 2,3. Entre outras características, essa condição foi associada com a síndrome de burnout, que retrata um indivíduo que lida com o público (a maioria médicos, enfermeiros, profissionais da saúde em geral e professores) e que se mostra desmotivado, pouco compreensivo, com tratamento distante e desumanizado para com os pacientes, culpando-os dos problemas de que padecem 3 .

Desde então, vários autores, como Maslach (1976), Cherniss (1980), Pines (1989), dentre outros, expuseram suas teorias e definições sobre o burnout ${ }^{2}$. A proposta de Maslach foi a de maior impacto e aceitação acadêmica, definindo-a como uma síndrome de cansaço emocional, despersonalização e baixa realização pessoal, que pode ocorrer entre indivíduos cujo trabalho requer contato com pessoas, principalmente quando essa atividade é considerada de ajuda (médicos, enfermeiros, professores) 2,3,4,5,6,7,8,9,10 11,12 . Não se resumindo apenas aos trabalhadores 
da saúde e profissionais de educação, o burnout também ocorre em indivíduos cujas profissões os expõem à tensão e estresse intensos, como policiais, contadores, corretores de bolsa, diretores ou executivos de empresas, controladores de tráfego aéreo ${ }^{13}$, treinadores e desportistas, sendo inclusive estudado fora do âmbito profissional (donas-de-casa, por exemplo) 7. Christina Maslach, Cary Cherniss e Alaya Pines foram os estudiosos que popularizaram o conceito da síndrome e o legitimaram como uma importante questão ocupacional e social 2,8. Maslach \& Goldenberg 5 (p. 63) também afirmam ser o burnout "um ponto final particularmente trágico para profissionais que começaram a trabalhar com expectativas positivas, entusiasmo e uma dedicação para ajudar as pessoas".

De modo geral, "pode-se definir o burnout como um transtorno adaptativo crônico associado às demandas e exigências laborais, cujo desenvolvimento é insidioso e freqüentemente não reconhecido pelo indivíduo, com sintomatologia múltipla, predominando o cansaço emocional" 3 (p. 177). Além desta última característica, e como já mencionado, outras duas compõem o quadro bem definido da síndrome: despersonalização e baixa realização pessoal 2,3,5,12,14,15. O cansaço emocional é considerado o traço inicial, podendo a manifestação ser física, psíquica ou uma combinação das duas. É descrito como o núcleo da síndrome e a sua manifestação mais óbvia 16 . A despersonalização, caracterizada pela insensibilidade emocional do profissional, com prevalência de condutas cínicas e de dissimulação afetiva, é uma reação imediata após a instalação do cansaço 2,3,6,8,10,11,16. A baixa realização pessoal faz menção a uma auto-avaliação negativa associada à insatisfação e desânimo com o trabalho, com sentimentos de que este não vale a pena 2,6,8,10. Segundo Maslach \& Goldenberg 5, o processo avança de modo seqüencial, em que a ocorrência de um componente precipita o próximo: o cansaço emocional acontece primeiro e conduz ao desenvolvimento de despersonalização. Atualmente, considera-se que a redução da realização pessoal se desenvolve separadamente.

Enfermeiros, técnicos e auxiliares de enfermagem fazem parte de uma profissão caracterizada por ter, em sua essência, o cuidado e por grande parte da carga de trabalho ser o contato direto com pacientes e familiares. Do ponto de vista da organização do trabalho, a indefinição do papel profissional; a sobrecarga de trabalho freqüentemente justificada por falta de pessoal e estimulada pelo pagamento de horas-extras; a falta de autonomia e autoridade na tomada de decisões, entre outras, geram um estado de estresse crônico, identificando-se como uma das profissões de maior incidência de burnout 2,4,17. Segundo a Health Education Authority, a enfermagem é classificada como a quarta profissão mais estressante no setor público 2,17. A deterioração na qualidade de serviços de instituições de saúde e os altos índices de absenteísmo dos profissionais dessa área são algumas das conseqüências desse quadro, todas fortemente relacionadas com a alta taxa de incidência que caracteriza a síndrome ${ }^{4}$. Em relação ao sexo, estudos mostram que a prevalência de burnout é maior em homens 4 . Apesar disso, nas atividades de enfermagem, consideradas tipicamente femininas, a incidência da síndrome também é bastante alta 4,10 .

A despeito da situação apresentada anteriormente, poucas foram as pesquisas realizadas no Brasil que procuravam investigar os problemas de saúde que esses trabalhadores enfrentam, associando-os com as características dessa enfermidade.

Trabalhos relacionando a síndrome de burnout com o trabalho da saúde são mais comuns em países da América do Norte, Europa e parte da América Latina, mas, ainda assim, não estudam especificamente os trabalhadores da área da enfermagem. Enfermeiros, técnicos e auxiliares de enfermagem, por serem os profissionais da saúde que mais tempo passam em contato com o paciente e com seus familiares dentro do ambiente de trabalho, constituem um grupo com grande predisposição ao desenvolvimento da síndrome. As implicações para a área da saúde devido a esse fato são relevantes, já que a alta freqüência de faltas ao trabalho, pedidos de licença, abandono do emprego e deterioração da qualidade dos serviços têm impacto negativo sobre a efetividade da atenção oferecida aos pacientes.

Esta pesquisa se propôs a determinar a prevalência da síndrome de burnout nos enfermeiros, técnicos e auxiliares de enfermagem em um hospital de grande porte localizado na Região Sul do Brasil, o Hospital Nossa Senhora da Conceição, localizado em Tubarão, Santa Catarina, traçando o perfil dos trabalhadores mais propensos a desenvolver burnout. Foram feitas associações deste quadro com os dados sócio-culturais dos entrevistados, verificandose a distribuição da síndrome entre os diferentes serviços do hospital.

\section{Métodos}

A pesquisa realizada, com abordagem quantitativa, foi concebida como um estudo epidemiológico de tipo transversal. 
População e amostragem foram selecionadas entre os enfermeiros, técnicos e auxiliares de enfermagem do Hospital Nossa Senhora da Conceição. Os diversos setores do hospital foram avaliados de acordo com a natureza das atividades desenvolvidas, a capacidade instalada e número de empregados, visando à organização de grupos homogêneos de trabalhadores para participação no estudo. O número de funcionários desses setores totalizou 279 profissionais, segundo o Departamento de Recursos Humanos do hospital em estudo, sendo 20 enfermeiros, 132 técnicos de enfermagem e 127 auxiliares de enfermagem. Os diversos serviços do hospital foram organizados em cinco grupos diferentes, sendo os setores 5, 7, 8, 9, 12, o Alojamento Conjunto e a Ala São Vicente, agrupados sob a denominação "setores". Os demais grupos foram a UTI Adulto, UTI Neonatal, Centro Obstétrico e Emergência. Com a distribuição dos trabalhadores agregada desse modo, a amostra foi selecionada de forma aleatória estratificada por conglomerados, sendo cada setor do hospital considerado um conglomerado.

O Hospital Nossa Senhora da Conceição é uma instituição centenária, de direito privado e sem fins lucrativos, cuja entidade mantenedora é a Sociedade Divina Providência. O estabelecimento tem a função de centro de referência regional para procedimentos de média e alta complexidade ambulatorial e hospitalar, sendo grande parte dos seus serviços utilizados pelo Sistema Único de Saúde (SUS). Atualmente é o maior hospital do estado em número de leitos (total de 400) e emprega mais de 720 funcionários e 135 médicos 18 .

Foram incluídos na amostra pesquisada todos os enfermeiros, técnicos e auxiliares de enfermagem com vínculo formal com a instituição Hospital Nossa Senhora da Conceição, desde que lotados nos setores selecionados para o estudo, cuja tarefa estivesse associada diretamente ao cuidado com pacientes e que aceitaram participar da pesquisa. Foram excluídos todos os trabalhadores que não se enquadraram nos critérios mencionados.

A coleta dos dados foi realizada no período de agosto a novembro de 2007, em entrevistas individuais, no próprio hospital. Os participantes foram abordados dentro do seu horário de trabalho, de acordo com a disponibilidade para participar do estudo.

Em relação à seleção dos instrumentos para a avaliação da síndrome, optamos pela aplicação do Maslach Burnout Inventory (MBI), elaborado por Christina Maslach e Susan Jackson em 1978, que, de acordo Gil-Monte \& Peiró 19, é o instrumento mais utilizado para avaliar o burnout, independentemente das características ocupacionais da amostra e de sua origem $3,5,7,8,16$. Esse instrumento avalia as três dimensões da síndrome - cansaço emocional, despersonalização e realização profissional -, desconsiderando antecedentes prévios e conseqüências de seu processo ${ }^{8}$. O MBI tem várias versões para aplicação em situações de trabalho específicas, tais como o MBI-Human Services Survey (MBI-HSS), o MBI-Educators Survey (MBI-ES) e o MBI-General Survey (MBI-GS) 16.

Os sujeitos da pesquisa responderam, inicialmente, a questionário com perguntas de abordagem sócio-cultural, organizado pelos autores, seguido do MBI, em sua versão adaptada e validada ao português por Tamayo 20 . Trata-se de um questionário de 22 perguntas, com cinco opções de resposta (escala Likert de 1 a 5), que engloba os três aspectos fundamentais da síndrome de burnout. Os detalhes da organização do MBI estão apresentados na Tabela 1 .

Para analisar a prevalência da síndrome no seu conjunto (as três dimensões agrupadas), foram seguidos os critérios apresentados por Ramirez et al. 21 e Grunfeld et al. 22. Ramirez et al. 21 definem burnout quando se encontram altas pontuações em cansaço emocional e despersonalização e baixas pontuações na subescala realização pessoal. Grunfeld et al. ${ }^{20}$, por sua vez, consideram o diagnóstico de burnout

Padrão de pontuação para diagnóstico das dimensões da síndrome de burnout pelo Maslach Burnout Inventory (MBI) 10,13.

\begin{tabular}{|c|c|c|c|c|}
\hline \multirow[t]{2}{*}{ Dimensões } & \multirow[t]{2}{*}{ Questões } & \multicolumn{3}{|c|}{ Padrão para pontuação } \\
\hline & & Nível alto & Nível médio & Nível baixo \\
\hline Cansaço emocional & $1,2,3,6,8,13,14,16$ e 20 & $\geq 27$ & $19-26$ & $<19$ \\
\hline Despersonalização & $5,10,11,15$ e 22 & $\geq 10$ & $6-9$ & $<6$ \\
\hline Realização pessoal & $4,7,9,12,17,18,19$ e 21 & $\leq 33$ & $34-39$ & $\geq 40$ \\
\hline
\end{tabular}


quando o indivíduo pontua nível alto em cansaço emocional ou despersonalização, ou nível baixo em realização pessoal.

A partir da quantificação da população-alvo e a definição da freqüência esperada de síndrome de burnout em aproximadamente $20 \%$ com erro $\alpha=5 \%$, a amostra calculada no módulo Statcalc do Epi Info 6.04 (Centers for Disease Control and Prevention, Atlanta, Estados Unidos) foi de 131 indivíduos.

Para a tabulação, processamento e a análise dos dados das questões objetivas foram utilizados os programas Epidata 3.1 (Epidata Association, Odense, Dinamarca), Epi Info 6.04 e SPSS 14.0 (SPSS Inc., Chicago, Estados Unidos).

As variáveis quantitativas foram descritas por meio de medidas de tendência central e dispersão. As variáveis qualitativas foram descritas por meio de razões e proporções.

As diferenças nas proporções foram testadas pelo teste de qui-quadrado $\left(\chi^{2}\right)$ ou prova exata de Fisher, quando adequado.

As diferenças nas médias de variáveis quantitativas foram testadas pelo teste $t$ de Student ou Kruskall-Wallis, quando cabível.

Para testar o efeito independente das variáveis nos desfechos de alto risco para burnout em cada um dos domínios, foi utilizada a regressão logística através do programa SPSS 14.0.

O projeto de pesquisa foi elaborado de acordo com o que preconiza a Resolução $n^{\circ}$. 196/96 do Conselho Nacional de Saúde e segundo as normas do Comitê de Ética e Pesquisa (CEP) da Universidade do Sul de Sana Catarina (UNISUL), pelo qual foi avaliado e aprovado à luz dos princípios éticos de autonomia, beneficência, não maleficência, justiça e equidade. Foi solicitado o consentimento livre e esclarecido para todos os indivíduos participantes.

\section{Resultados}

A taxa de resposta obtida foi de $100 \%$, uma vez que todos trabalhadores abordados concordaram em responder à pesquisa. Foram coletados 151 questionários, correspondendo a 54,12\% dos 279 empregados cadastrados no Departamento de Recursos Humanos do Hospital Nossa Senhora da Conceição, nos setores selecionados para o estudo. Os auxiliares de enfermagem responderam em menor percentual (Tabela 2) por esta função estar gradualmente sendo substituída por profissionais com formação técnica em enfermagem.

Os resultados do questionário sócio-cultural estão descritos na Tabela 2.
Tabela 2

Dados sócio-culturais dos trabalhadores entrevistados. Hospital Nossa Senhora da Conceição, Tubarão, Santa Catarina, Brasil.

\begin{tabular}{|c|c|}
\hline Variáveis & $\begin{array}{c}\text { Resultados } \\
\text { n (\%) }\end{array}$ \\
\hline Idade & $\begin{array}{c}\text { Média: } 31,75 \text { anos ( } \pm 8,25) \\
\text { (variação: } 20-55 \text { anos) }\end{array}$ \\
\hline \multicolumn{2}{|l|}{ Gênero } \\
\hline Feminino & $137(90,7)$ \\
\hline Masculino & $14(9,3)$ \\
\hline \multicolumn{2}{|l|}{ Estado civil } \\
\hline Solteiro (a) & $63(41,7)$ \\
\hline \multicolumn{2}{|l|}{ Casado(a) ou } \\
\hline com companheiro(a) & $77(51,0)$ \\
\hline Divorciado(a)/Viúvo(a) & $11(7,3)$ \\
\hline \multicolumn{2}{|l|}{ Filhos } \\
\hline 0 & $64(42,4)$ \\
\hline 1 & $39(25,8)$ \\
\hline 2 ou mais & $48(31,8)$ \\
\hline \multicolumn{2}{|l|}{ Cargo ocupado } \\
\hline Enfermeiro(a) & $15(9,9)$ \\
\hline Técnico(a) de enfermagem & $106(70,2)$ \\
\hline Auxiliar de enfermagem & $30(19,9)$ \\
\hline \multicolumn{2}{|l|}{ Setor onde trabalha } \\
\hline Setores & $73(48,4)$ \\
\hline UTI Adulto & $35(23,2)$ \\
\hline UTI Neonatal & $18(11,9)$ \\
\hline Centro Obstétrico & $11(7,3)$ \\
\hline Emergência & $14(9,2)$ \\
\hline Tempo de profissão (anos) & Média: 6,7 anos \\
\hline $1-10$ & $116(76,8)$ \\
\hline $11-20$ & $27(17,9)$ \\
\hline Acima de 20 & $8(5,3)$ \\
\hline \multicolumn{2}{|l|}{ Hobby } \\
\hline Sim & $124(82,1)$ \\
\hline Não & $27(17,9)$ \\
\hline \multicolumn{2}{|l|}{ Licença no trabalho por } \\
\hline \multicolumn{2}{|l|}{ motivo de saúde } \\
\hline Sim & $21(13,9)$ \\
\hline Não & $130(86,1)$ \\
\hline
\end{tabular}

Os valores médios obtidos nas três dimensões da síndrome de burnout, segundo o MBI, foram: 17 pontos em cansaço emocional, indicando nível baixo; 7,79 pontos em despersonalização, indicando nível médio; e 36,6 pontos em realização pessoal, nível médio (Tabela 3).

Quanto aos níveis individuais de cada dimensão, em cansaço emocional, $62,2 \%$ pontuaram baixo, $31,8 \%$, médio, e $6 \%$ pontuaram alto; em despersonalização, 25,8\% pontuaram baixo, 
Resultados do Maslach Burnout Inventory (MBI) entre os trabalhadores entrevistados. Hospital Nossa Senhora da Conceição, Tubarão, Santa Catarina, Brasil.

\begin{tabular}{lc}
\hline Dimensões & $\begin{array}{c}\text { Resultados } \\
\mathbf{n}(\%)\end{array}$ \\
\hline Cansaço emocional & Média: $17( \pm 5,39)$ \\
Baixo & $94(62,2)$ \\
Médio & $48(31,8)$ \\
Alto & $9(6,0)$ \\
Médio e alto & $57(37,8)$ \\
Despersonalização & Média: $7,79( \pm 2,99)$ \\
Baixo & $39(25,8)$ \\
Médio & $79(52,3)$ \\
Alto & $33(21,9)$ \\
Médio e alto & $112(74,2)$ \\
Realização pessoal & Média: $36,6( \pm 5,8)$ \\
Baixo & $16(10,6)$ \\
Médio & $59(39,1)$ \\
Alto & $76(50,3)$ \\
Baixo e médio & $75(49,7)$ \\
Grunfeld et al. 22 & $54(35,7)$ \\
Ramirez et al. 21 & $0(0,0)$ \\
\hline
\end{tabular}

52,3\%, médio, e 21,9\% pontuaram alto; em realização pessoal, 10,6\% pontuaram baixo, 39,1\%, médio, e 50,3\%, alto.

Ao analisar os dados referentes à idade com as três dimensões do MBI, encontrou-se que a prevalência encontrada de médio ou alto nível de cansaço emocional entre os trabalhadores com idade acima de 35 anos foi mais que o dobro, em comparação aos com idade de 25 anos ou menos $(\mathrm{p}=0,02)$. Os maiores níveis de realização pessoal foram encontrados na faixa entre 26 e 35 anos, sendo a prevalência 50\% maior em relação aos com 25 anos ou menos ( $\mathrm{p}<0,05)$.

A maioria dos entrevistados, no que se refere ao gênero, apresentou níveis baixos em cansaço emocional. Destes, 86,2\% eram mulheres e 13,8\%, homens. Dos que apresentaram níveis médios, novamente as mulheres compunham o grupo maior, representando 97,9\% deste. Nenhum dos entrevistados masculinos pontuou alto nesta dimensão $(p=0,04)$. Há que se considerar a alta porcentagem de participação feminina no estudo $(90,7 \%)$. Não foram encontradas diferenças nas demais dimensões.

Na dimensão cansaço emocional, em relação ao estado civil, a maioria dos participantes $(62,3 \%)$ obteve baixa pontuação, sendo o grupo de solteiros o menos propenso aos sintomas dessa dimensão (50\%). Quanto ao grupo de casados/divorciados/viúvos, os solteiros apresentaram menor prevalência de níveis moderado e alto nessa dimensão (razão de prevalência $\mathrm{RP}=0,55$; intervalo de $95 \%$ de confiança $-\mathrm{IC} 95 \%$ : 0,34-0,88; $\mathrm{p}=0,008)$.

Na variável quantidade de filhos, analisando o cansaço emocional ( $\mathrm{p}<0,01$ ), foi verificado que, dos $62,3 \%$ que obtiveram baixa pontuação, $50 \%$ não tinham filhos, $24,5 \%$ tinham um filho e $25,6 \%$ tinham dois ou mais filhos. Analisando a despersonalização ( $\mathrm{p}=0,02)$, encontrou-se que $52,3 \%$ apresentaram nível médio de pontuação, a maioria dos quais tinham filhos $(60,8 \%)$. As pessoas sem filhos foram associadas a menores níveis de cansaço emocional e despersonalização $(\mathrm{p}=0,01)$.

Em nosso trabalho, não foram encontradas associações estatisticamente significativas entre as dimensões de burnout e as variáveis: cargo desempenhado, setor no qual trabalha, tempo de profissão, distração ou hobby e solicitação de licença no último ano.

Após o ajuste pela regressão logística, a única variável associada à alta pontuação na dimensão cansaço emocional foi o tempo de profissão acima de cinco anos. Nos domínios de despersonalização, realização pessoal e risco geral de burnout, nenhuma das variáveis do modelo de regressão logística esteve associada de forma estatisticamente significativa com risco alto para a síndrome (Tabela 4).

Para estabelecer a prevalência da síndrome na amostra estudada, utilizando os critérios estabelecidos por Grunfeld et al. 22, encontramos um total de 54 indivíduos (35,7\%) com diagnóstico de burnout. A solicitação de licença do trabalho por motivo de saúde no último ano apresentou correlação $(\mathrm{p}=0,02)$ para esse grupo de trabalhadores.

Utilizando os critérios de Ramirez et al. 21, não houve diagnóstico de burnout entre os entrevistados.

A distribuição dos trabalhadores pelos conglomerados de pesquisa foi semelhante à dos casos encontrados (Figura 1), não tendo sido encontrada associação estatística entre o diagnóstico da síndrome e o processo de trabalho específico de cada unidade assistencial.

\section{Discussão}

As pesquisas sobre burnout cresceram em complexidade à medida que se aprofundaram na identificação e avaliação de suas causas e determinantes. 
Resultados da regressão logística.

\begin{tabular}{lcccc}
\hline Variáveis independentes & \multicolumn{2}{c}{ Odds ratio (IC95\%) } \\
& Emocional & Despersonalização & Realização & Risco burnout \\
\hline Gênero feminino & $0,15(0,18-1,29)$ & $0,65(0,18-2,35)$ & $0,31(0,06-1,60)$ & $1,42(0,43-4,71)$ \\
Idade > 40 anos & $1,77(0,66-4,72)$ & $1,02(0,32-3,22)$ & $2,47(0,37-16,53)$ & $1,39(0,55-3,50)$ \\
Estado civil (solteiro, divorciado, viúvo) & $1,33(0,60-2,93)$ & $0,81(0,35-1,91)$ & $1,52(0,42-5,47)$ & $0,67(0,32-1,40)$ \\
Sem filhos & $1,66(0,69-3,99)$ & $2,52(0,98-6,44)$ & $3,92(0,83-18,58)$ & $1,85(0,83-4,11)$ \\
Cargo (enfermeiro e técnico) & $0,86(0,35-2,12)$ & $1,19(0,45-3,12)$ & $0,25(0,03-2,05)$ & $0,78(0,34-1,81)$ \\
Tempo profissão (mais de cinco anos) & $0,33(0,14-0,75) *$ & $1,07(0,42-2,69)$ & $1,06(0,27-4,17)$ & $0,85(0,38-1,90)$ \\
Hobby & $0,70(0,27-1,79)$ & $0,58(0,18-1,90)$ & $0,37(0,04-3,26)$ & $0,52(0,21-1,27)$ \\
Licença & $0,60(0,21-1,68)$ & $0,60(0,22-1,68)$ & $0,51(0,06-4,42)$ & $0,77(0,29-2,03)$ \\
\hline
\end{tabular}

*Valor estatisticamente significante $(p<0,05)$.

\section{Figura 1}

Distribuição dos casos de burnout de acordo com os critérios de Grunfeld et al. 22, segundo local de trabalho. Hospital Nossa Senhora da Conceição, Tubarão, Santa Catarina, Brasil.

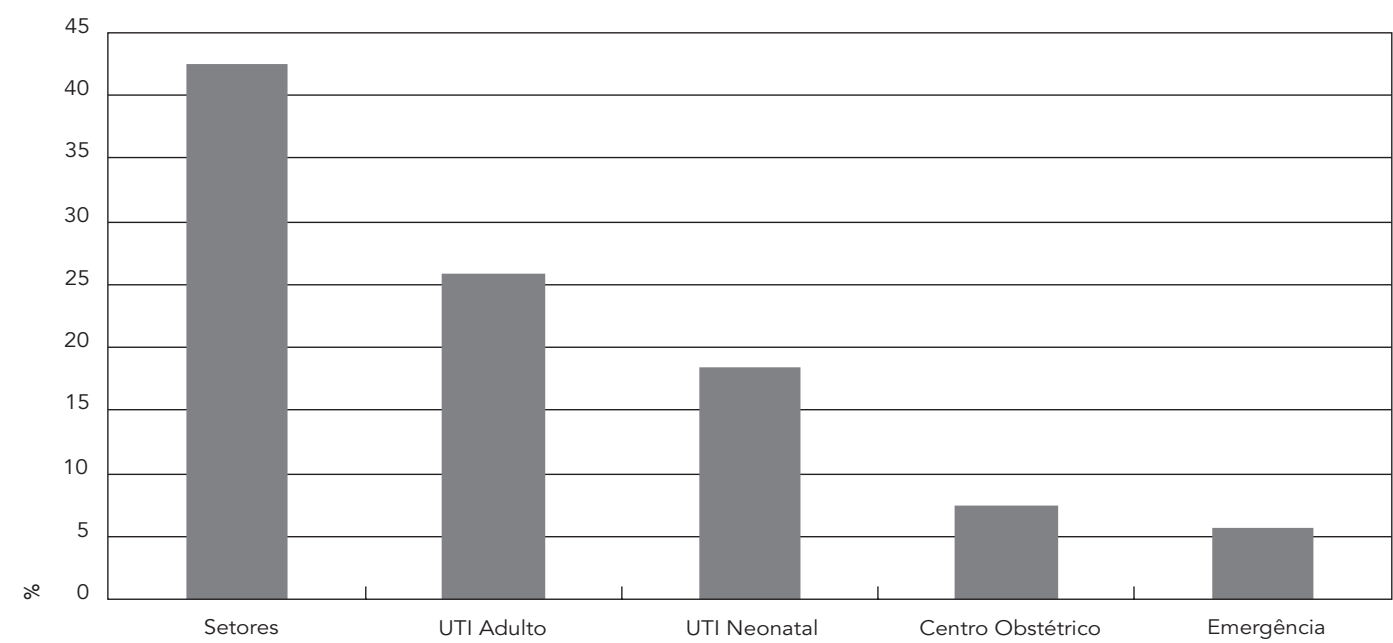

O instrumento utilizado em nosso estudo, o MBI, desde a sua criação por Christina Maslach e Susan Jackson (1978), é o meio mais utilizado para avaliar o burnout, contudo, ao longo dos anos, sofreu modificações em sua estrutura. Originalmente composto por 47 perguntas, hoje conta com 22 23. As traduções da língua original, inglês, foram testadas e validadas em diferentes países. Tamayo 20, em sua dissertação de mestrado, adaptou o MBI para a língua portuguesa, aplicando o questionário a enfermeiros e auxi- liares de enfermagem em hospitais do Distrito Federal, encontrando valores semelhantes aos da versão americana 24 . No atual estado da arte do burnout, o MBI apresenta-se como uma escala fidedigna e válida, independentemente de onde a síndrome seja estudada 8 .

Apesar disso, atualmente estão sendo propostos novos métodos de avaliação que superam as deficiências encontradas no MBI. Exemplo disto é o consenso atual em considerar a realização pessoal mais como um fator interdependen- 
te do que uma dimensão interna da síndrome (atualmente considera-se o baixo grau de realização pessoal uma conseqüência do desgaste profissional). Esses instrumentos estão sendo desenvolvidos especialmente na Europa e supõem um novo conceito da síndrome. São exemplos: o Oldenburg Burnout Inventory (OLBI), que inclui somente duas dimensões (cansaço emocional e falta de compromisso com o trabalho) e o Cuestionario para la Evaluación del Síndrome de Quemarse en el Trabajo (Espanha) 25.

Nosso estudo foi desenvolvido junto a um grupo de trabalhadores cujo perfil médio é o de profissional do sexo feminino, técnico de enfermagem, entre 26 e 35 anos, casado, sem filhos e com menos de dez anos de profissão.

A maior participação de mulheres no estudo $(90,7 \%)$ pode ser explicada, segundo Gil-Monte ${ }^{4}$, pela própria composição feminina dessa categoria profissional, historicamente associada à atividade caritativa religiosa (feminização do cuidado), ainda que se tenha observado aumento no número de homens nos últimos anos. A mudança desse perfil pode estar associada à acessibilidade ao emprego, curta duração dos estudos e inclusão destes em catálogos de titulação universitárias.

As médias de pontuação das dimensões cansaço emocional, despersonalização e realização pessoal do MBI encontradas no estudo (17; 7,79 e 36,6 respectivamente) diferem das encontradas por Tucunduva et al. 6 (21,4; 9,7 e 42,0 respectivamente), que utilizou os mesmos pontos de corte empregados em nosso estudo, descritos na metodologia. Resultados análogos foram encontrados por Albaladejo et al. 26 nas dimensões cansaço emocional e despersonalização (pontos de corte semelhantes), com médias obtidas de 19,61 e 8,37 respectivamente. Gil-Monte 4 também encontrou resultados semelhantes em despersonalização, apresentando média de 6,38.

Por não haver consenso na literatura para a interpretação do MBI, publicações apresentam critérios diferentes no intuito de classificar e diagnosticar o burnout. Grunfeld et al. 22 definem a estafa profissional como a presença em nível grave (pontuação em nível baixo) de uma das dimensões; Ramirez et al. ${ }^{21}$ e Albaladejo et al. 26 defendem que o diagnóstico é feito quando se evidenciam as três dimensões em nível grave. Por isso, como afirmou Freudenberger (1980, apud Albaladejo et al. 26), a inclusão dos três aspectos ou dimensões do burnout enfatiza a complexidade do mesmo.

Em nossa pesquisa, considerando Grunfeld et al. 22, 35,7\% dos trabalhadores apresentaram burnout, número inferior ao apresentado por Tucunduva et al. 6 em seu estudo, que encon- trou 52,3\% dos entrevistados com a síndrome. O perfil do grupo de trabalhadores que apresentou burnout foi semelhante ao perfil médio do conjunto dos trabalhadores pesquisados: sexo feminino, técnico de enfermagem, entre 26 e 35 anos, casado, sem filhos e com menos de dez anos de profissão. Encontramos uma associação significativa entre os trabalhadores com maiores níveis da síndrome e pedidos de licença para tratamento de saúde, corroborando indicação de Gil-Monte 4, que observou número maior de faltas no trabalho entre trabalhadores com burnout. A ausência de casos segundo critérios de Ramirez et al. 21 deve-se ao fato de que este autor utiliza uma classificação mais rígida, como já foi mencionado. Outras pesquisas utilizando os critérios desse autor também apresentaram baixos índices de burnout 6 .

Globalmente, os profissionais do Hospital Nossa Senhora da Conceição se enquadraram no intervalo entre risco baixo e médio de desenvolver burnout.

Confirmando a literatura, os trabalhadores com idade abaixo de 26 anos mostraram-se menos realizados, em comparação com os mais velhos. Isso pode ser explicado pelo fato de que, no começo da profissão, esses profissionais se encontram em um período de sensibilização, quando a transição das expectativas idealistas para a realidade da prática cotidiana mostra que as primeiras nem sempre se realizam como prometido ou esperado ${ }^{23}$. Apesar disso, a associação da síndrome com o tempo de profissão (mais de cinco anos) sugere que a exposição prolongada a condições de trabalho estressantes e subvalorizadas pode estar relacionada ao surgimento e ao agravamento dos sintomas.

Gil-Monte 4 (p. 5) afirma que, quanto ao gênero, "a tendência geral dos resultados é obter diferenças significativas em função do sexo nos níveis de despersonalização, no sentido de que os homens pontuam significativamente mais alto que mulheres nesta dimensão". Em nosso estudo, não foi observada relação significativa entre a variável gênero e despersonalização, com resultados apresentando valores muito próximos para os dois sexos (apesar de as mulheres pontuarem mais alto). Entretanto, como a amostra foi formada predominantemente por mulheres, o processo de burnout influenciado pelo gênero pode estar mascarado 4 . Analisando o burnout como uma síndrome (e não como variáveis independentes), as mulheres trabalhadoras da saúde compõem o grupo mais vulnerável, fato explicado, talvez, pelas cargas de trabalho excessivas concomitantes com as da vida familiar 23 .

No que se refere ao estado civil, pessoas solteiras são mais propensas a apresentar a síndro- 
me 10,15, embora não haja unanimidade quanto a essa relação 23 . Em nossa pesquisa, os solteiros foram os que se mostraram menos emocionalmente cansados, compondo metade do grupo que pontuou baixo nessa dimensão. Dentre os que apresentaram risco médio em cansaço emocional, o grupo de sujeitos casados foi o que mais pontuou. Neste aspecto, esses resultados não confirmam a literatura, que indica que as pessoas casadas ou com companheiro(a) estável experimentam sentimentos de responsabilidade familiar e maior resistência à síndrome, por uma associação com a capacidade de enfrentar problemas emocionais 15 .

De igual modo, complementando as variáveis associadas ao núcleo familiar, indivíduos com filhos apresentaram menores taxas de burnout em relação aos trabalhadores sem filhos, possivelmente devido à associação da maternidade/paternidade com maior responsabilidade, maturidade, estabilidade e expectativas mais realistas 23. Encontramos significância estatística nas dimensões cansaço emocional e despersonalização em relação à variável número de filhos, tendo sido verificados resultados diferentes dos já publicados $(\mathrm{p}<0,05)$. Agregando os sujeitos pesquisados em grupos com e sem filhos, os que mais apresentaram médio risco de cansaço emocional foram os do segundo grupo, com prevalência de quase $70 \%$. Entre os com nível de cansaço emocional baixo, os dois grupos apresentaram valores equivalentes. Em despersonalização, aproximadamente $60 \%$ dos indivíduos com nível médio tinham filhos e, entre os com nível alto, $70 \%$.

Apesar de não havermos encontrado significância na dimensão realização pessoal $(p=0,21)$ em relação ao número de filhos, vale ressaltar que $90 \%$ dos pesquisados mostraram-se moderadamente ou altamente realizados, dentre os quais $54 \%$ tinham filhos.

Contudo, como afirmaram Gil-Monte \& Peiró 19 , o estado civil e a maternidade/paternidade não são os únicos fatores de proteção em relação ao burnout, contribuindo para tal também o apoio oferecido por parte dos familiares.

\section{Conclusão}

Em virtude da diversidade de formas com que os estudos acadêmicos vêm assumindo a avaliação da síndrome de burnout, especialmente no que diz respeito à interpretação do MBI (pontos de corte para estabelecer os níveis alto, moderado e baixo das dimensões, assim como o modo de interpretação do instrumento para definição do diagnóstico), não foi possível estabelecer compa- rações mais consistentes entre os resultados de nossa pesquisa com os de outros trabalhos pesquisados na literatura. Em sua maioria, os dados apresentados pela literatura disponível revelam números e classificações para trabalhadores de outros países, principalmente norte-americanos. Em contrapartida, verifica-se que um número reduzido de pesquisas sobre o assunto foi realizado no Brasil, fator que tende a se modificar, já que o tema adquiriu certa visibilidade no meio acadêmico e novos trabalhos estão em andamento em nosso meio.

A pesquisa aqui apresentada verificou que, no que diz respeito às dimensões isoladas pesquisadas pelo $\mathrm{MBI}$, os trabalhadores de enfermagem do Hospital Nossa Senhora da Conceição indicaram níveis baixos ou médios para burnout em todas elas. Salientamos que mais da metade dos trabalhadores entrevistados assinalou nível de cansaço emocional baixo e 50\% assinalaram níveis de realização pessoal altos. Apesar disso, a prevalência da síndrome de burnout encontrada entre os trabalhadores pesquisados (critério de Grunfeld et al. 22) foi relativamente alta, uma vez que mais de um terço deles $(35,7 \%)$ apresentou pelo menos uma dimensão da síndrome em níveis críticos.

Grande parte das associações estudadas não obteve significância estatística, o que reduziu a possibilidade de avaliação de características pessoais associadas à síndrome. Este achado sugere que as condições de trabalho como um todo, mais do que as características isoladas dos trabalhadores ou do ambiente de trabalho, são responsáveis pela emergência dos sintomas do burnout. Além disso, várias associações encontradas afrontaram os padrões indicados pela literatura consultada, sugerindo a necessidade de novos estudos para confirmação dos padrões nos quais a síndrome se insere.

A presença maciça das mulheres na amostra estudada confirma o estereótipo da profissão de enfermagem (em cuja essência encontra-se o cuidado) como ocupação predominantemente feminina. Ademais, para muitas delas as responsabilidades profissionais se somam com as responsabilidades da vida diária, ocasionando um acúmulo de elementos estressores que podem levar ao burnout, apesar de não termos encontrado essa associação de forma inequívoca.

Nosso estudo pesquisou um grupo de trabalhadores composto majoritariamente por mulheres, entre 26 e 35 anos, casadas, sem filhos e com menos de dez anos de profissão, perfil semelhante ao encontrado entre os trabalhadores que apresentaram burnout.

O coeficiente de prevalência encontrado entre esse grupo de profissionais pode ser conside- 
rado alto, pois afeta a qualidade de vida de mais de um terço deles. Portanto, a identificação e o controle dos fatores geradores dos sinais e sintomas apresentados pelos trabalhadores entrevistados podem contribuir para a promoção da sua saúde individualmente, da saúde do grupo de trabalhadores de enfermagem (que estão sujeitos às mesmas condições de trabalho) 10,16 e para a melhoria da qualidade dos serviços prestados.

O Manual de Doenças Relacionadas ao Trabalho ${ }^{9}$, do Ministério da Saúde, indica que, quando o diagnóstico de burnout é confirmado, este deve ser abordado como evento sentinela e indica investigação mais criteriosa da situação do trabalho (Classificação Internacional de Doenças, 10a revisão - CID-10: Z56.3, Z56.6).

\section{Resumo}

A síndrome de burnout é um transtorno adaptativo crônico que acomete trabalhadores e caracteriza-se por três expressões de sofrimento psíquico: cansaço emocional, despersonalização e baixa realização pessoal. Por meio de pesquisa quantitativa com delineamento transversal, foram aplicados questionários com dados sócio-culturais e o Maslach Burnout Inventory em 151 trabalhadores do serviço de enfermagem, correspondendo a 54,1\% desta categoria profissional em hospital geral de grande porte localizado em Tubarão, Santa Catarina, Brasil. Foram encontradas pontuações médias em cansaço emocional (17), despersonalização $(7,79)$ e em realização pessoal $(36,6)$. Segundo o critério de Ramirez et al., não houve diagnóstico da síndrome. De acordo com os critérios de Grunfeld et al., 35,7\% dos entrevistados apresentaram burnout. $O$ perfil padrão do trabalhador com burnout encontrado pela pesquisa foi: cargo de técnico de enfermagem, sexo feminino, entre 26 e 35 anos, casado, sem filhos e com mais de cinco anos de profissão. Os locais de trabalho que concentraram maior número de trabalhadores com burnout foram os setores agrupados (42,6\%), a UTI (25,9\%) e a UTI Neonatal (18,5\%). Não houve associação estatística entre local de trabalho e burnout.

Esgotamento Profissional; Serviços de Enfermagem; Saúde do Trabalhador
Certamente outros estudos devem continuar desvendando as relações entre o trabalho e a saúde dos trabalhadores. A pesquisa realizada e aqui apresentada, ao indicar o sofrimento psíquico a que está submetido um grupo de trabalhadores da saúde do Município de Tubarão, explicita condições de trabalho que devem ser investigadas de forma mais consistente em busca de respostas que possam contribuir para a prevenção de novos casos, a recuperação dos já acometidos e, principalmente, a promoção de condições de vida e trabalho que garantam a saúde física e mental de todos os trabalhadores de saúde de forma permanente.

\section{Colaboradores}

D. S. Moreira contribuiu com o levantamento de bibliografia, coleta, análise, interpretação dos dados e redação do trabalho. R. F. Magnago participou do levantamento bibliográfico e revisão do artigo. T. M. Sakae colaborou na análise estatística, análise, interpretação dos dados e descrição dos resultados. F. R. L. Magajewski desenvolveu função de orientação, redação e revisão em todas as fases do trabalho. 


\section{Referências}

1. Freudenberger HJ. Staff burn-out. J Soc Issues 1974; 30:159-65.

2. Murofuse NT, Abranches SS, Napoleão AA. Reflexões sobre estresse e Burnout e a relação com a enfermagem. Rev Latinoam Enferm 2005; 13: 255-61.

3. Schwartzmann L. Estrés laboral, síndrome de desgaste (quemado), depresión: ¿estamos hablando de lo mismo? Cienc Trab 2004; 6:174-84.

4. Gil-Monte PR. Influencia del género sobre el proceso de desarrollo del síndrome quemarse por el trabajo (burnout) en profesionales de enfermería. Psicol Estud 2002; 7:3-10.

5. Maslach C, Goldberg J. Prevention of burnout: new perspectives. Appl Prev Psychol 1998; 7:63-74.

6. Tucunduva LT, Garcia AP, Prudente FV, Centofanti G, Souza CM, Monteiro TA, et al. Incidence of the burnout syndrome among Brazilian cancer physicians. Rev Assoc Med Bras 2006; 52:108-12.

7. Gil-Monte PR. Validez factorial de la adaptación al español del Maslach Burnout Inventory-General Survey. Salud Pública Méx 2002; 44:33-40.

8. Carlotto M, Câmara S. Análise fatorial do Maslach Burnout Inventory (MBI) em uma amostra de professores de instituições particulares. Psicol Estud 2004; 9:499-505.

9. Ministério da Saúde/Organização Pan-Americana da Saúde. Doenças relacionadas ao trabalho: manual de procedimentos para os serviços de saúde. Brasília: Ministério da Saúde; 2001. (Série A. Normas e Manuais Técnicos).

10. Gómez MMN, Dodino CN, Aponte CF, Caycedo CE, Riveros MP, Martínez MPM, et al. Relación entre perfil psicológico, calidad de vida y estrés asistencial en personal de enfermería. Revista Universitas Psychologica 2005; 4:63-75.

11. Alpi SV, Flórez LA. El síndrome del Burnout en una muestra de auxiliares de enfermería: un estudio exploratorio. Revista Universitas Psychologica 2004; 3:35-45.

12. Iacovides A, Fountoulakis KN, Kaprinis S, Kaprinis G. The relationship between job stress, burnout and clinical depression. J Affect Disord 2003; 75:209-21.

13. Luna JMR. Síndrome de "burn out" ¿El médico de urgencias incansable? Revista Mexicana de Medicina de Urgencias 2002; 1:48-56.
14. Tena PS, Soriano JS, Bernal JS, Mulet EM, Muñoz CR, García MG, et al. Desgaste profesional en los médicos de atención primaria de Barcelona. Medifam 2002; 12:17-25.

15. Bianchini Matamoros M. El Síndrome del Burnout en personal profesional de la salud. Med Leg Costa Rica 1997; 13-14:189-92.

16. Maslach C, Schaufeli WB, Leiter MP. Job burnout. Annu Rev Psychol 2001; 52:397-422.

17. Stacciarini JM, Tróccoli BT. O estresse na atividade ocupacional do enfermeiro. Rev Latinoam Enferm 2001; 9:17-25.

18. Teixeira JW, Griebler L, Pereira C. 100 anos de amor pela vida. http://www.hnsc.org.br/ (acessado em 01/Nov/2007).

19. Gil-Monte PR, Peiró JM. Validez factorial del Malasch Burnout Inventory en una muestra multiocupacional. Psicothema 1999; 11:679-89.

20. Tamayo RM. Relação entre a síndrome de burnout e os valores organizacionais no pessoal de enfermagem de dois hospitais públicos [Dissertação de Mestrado]. Brasília: Instituto de Psicologia, Universidade de Brasília; 1997.

21. Ramirez AJ, Graham J, Richards MA, Cull A, Gregory WM. Mental health of hospital consultants: the effects of stress and satisfaction at work. Lancet 1996; 347:724-8.

22. Grunfeld E, Whelan TJ, Zitzelsberger L, Willan AR, Montesanto B, Evans WK. Cancer care workers in Ontario: prevalence of burnout, job stress and job satisfaction. CMAJ 2000; 163:166-9.

23. Martínez JCA. Aspectos epidemiológicos del síndrome de Burnout en personal sanitario. Rev Esp Salud Pública 1997; 71:293-303.

24. Borges LO, Argolo JCT, Pereira ALS, Machado EAP, Silva WS. A síndrome de burnout e os valores organizacionais: um estudo comparativo em hospitais universitários. Psicol Reflex Crit 2002; 15:189-200.

25. Moreno Jiménez B, Gálvez Herrer M, Garrosa Hernández E, Mingote Adán JC. Nuevos planteamientos en la evaluación del burnout: la evaluación específica del desgaste profesional médico. Aten Primaria 2006; 38:544-9.

26. Albaladejo R, Villanueva R, Ortega P, Astasio P, Calle ME, Domínguez V. Burnout syndrome among nursing staff at a hospital in Madrid. Rev Esp Salud Pública 2004; 78:505-16.

Recebido em 12/Ago/2008

Versão final reapresentada em 28/Jan/2009

Aprovado em 11/Fev/2009 